\title{
C. The phœnomena called by the name of Gravitation proved to be proximate effects of the orbicular and rotary motions of the earth
}

\section{Sir Richard Phillips}

To cite this article: Sir Richard Phillips (1817) C. The phœnomena called by the name of Gravitation proved to be proximate effects of the orbicular and rotary motions of the earth , Philosophical Magazine Series 1, 49:230, 430-442, DOI: 10.1080/14786441708637943

To link to this article: http://dx.doi.org/10.1080/14786441708637943

电 Published online: 27 Jul 2009.

Submit your article to this journal $\pi$

Џll Article views: 3

Q View related articles $\longleftarrow$ 


\section{The Phanomena of Gravitalion proved to be proximate}

of six. For some time they appeared plunged in a state of stupor and inertness; but scarcely had one hour elapsed before they showed themselves as vivacious and hearty as before.

So convincing were these experiments to one of the assistants, that he swallowed all the poison which could be extracted from four other large vipers, and was not in the least affected by it.

The following year the experiment was repeated on a crow after a fast of twelve hours, and it swallowed without injury the venom of six vipers.

In the month of October 1814 (continues the author of the memoir) I made seven large vipers discharge all their venom into one dish, and immediately dipped into it four small pieces of bread, which were swallowed by a pigeon. At first he appeared languid, but in a short time becaine as well as before. Some days after I inserted into one of his feet, as also into those of another pigeon, a small particle of very dry venom, which had been preserved for four months in a glass phial well corked up. Both of them soon gave manifest signs of being poisoned, and died at about the cnd of two hours.

Another pigeon swallowed all the venom which ten of the largest vipers could furnish, without presenting the least symptom of being poisoned.

Fontana alleged that the dry poison does not preserve its poisonous properties beyond nine months. This assertion was founded on experiments in which there was a possibility that the poison introduced into the wound might not have been retained, but expelled again with the flow of blood; and it is besides completely refuted by the following experiments of the author of this memoir. On poison preserved carefully for eighteen months, twenty-two months, and even twenty-six months, being introduced into the feet of several pigeons, they all died within half an hour or an hour. To secure the retention of the poison, the author immediately on inserting it stopped up the wound with a little lint. The result of these experiments completely demonstrates the error of Fontana's assertion, and proves that the poison of the viper prescrved with proper care may retain for many years its destructive properties.

C. The Phenomena called by the Name of Gravitation provel to be proximate Effects of the Orbicular and Rotary Motions of the Earth. By Sir Richand Phildips.

$I_{T}$ is the proper object and end of philosophy to iurestigate the mechanism of causes, and the means by which they produce 


\section{Fiffects of the Orbicular and Rotary Motion of the Earth. 431}

natural phænomena. For this purpose observers register fact, and philosophers infer the causes from the phænomena by a logical process of induction.

The design of the present essay is to determine the causes of all those phænomena, on which philosophical observers have hitherto conferred the name of Gravitation or Attraction, and which is vulgarly designated hy the name of Weight. Owing to what cause or causes does a body fall to the earth ?-Why does a projectile return to the earth ?-These are the questions which it is here proposed to answer.

The Newtonians, and all the modern schools of philosophy, have been unable to solve these problems; or, finding themselves unable, they have been unwilling to discuss them, or even tolerate their discussion : while the Theologians have been desirous of re-ferring this power to the proximate agency of the Deity. It is, however, the duty of genuine philosophers to persevere in spite of difficulties and obscurities; and of wise theologians to exalt their notions of the Deity by contemplating the sublime and simple mechanism of secondary causes.

In the present case, the phenomena consist in the apparent influence of one borly upon another, though they are not in contact, and though no visible, mechanical agency appears to exist between them; and in their approach to each other by certain laws of accelerated motion, as a result of apparently continued and reiterated forces.

What, however, are the circumstances in which the bodies so acting upon each other are placed, as in the case of a stone projected from, or falling to, the earth? The Earth is a globe of heterogeneous materials, moving round the sun in every year, at such distance, that its mean rate of motion, in round numbers, is 100,000 feet in a second of time; and the stone moves with the earth in the same orbit, partaking conjointly with it of the same mean motion of 100,000 fect in every second. Nor will any one doubt that, at the time the earth and atmosphere are performing this orbicular motion, they are also performing a rotary motion in every twenty-four hours, which rotary motion carries bodies on the earth's surface throughi a space, at the equator, of 1250 feet in a second, or one-eightieth of the orbicillar motion.

The whole earth, then, with all bodies upon the earth, and the atmosphere, are subject to these combined motions and forces; and, in this passive state, the questions are: by what law or laws the heterogeneous particulars are kept together? and how, if the positions are disturbed, those positions are restored? 


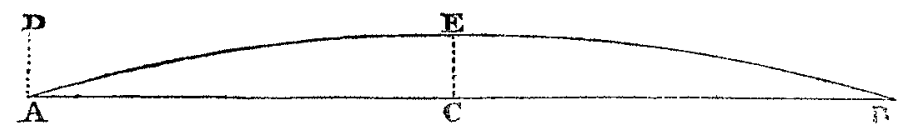

Suppose A to be a place on the earth's surface, from which, by muscular or explosive force, a stone is projected towards D, at sixteen feet and an inch above A. Suppose that a second of time elapses while the stone is ascending from $A$ to $D$, then it is evident that the point $A$ will in that second be carried forward, by the orbicular motion of the earth, 100,000 feet, or to $\mathrm{C}$; that is to say, the point $A$ will move 100,000 feet while the ball is ascending sixteen feet and an inch : consequently the ball will, in truth, not ascend in space from $A$ to $D$, but will be carried in an oblique line from $A$ to $E$, moving upwards as it proceeds. The two forces-that which carries it from $A$ to $C$, and that which carries it towards $D$, are as 100,000 to $16 \frac{1}{12}$, or as 6000 to I nearly.

The stone having arrived at $\mathrm{E}$, it is then known, by the phænomenon, to fall to the earth in a second of time; yet it does not fall through EC, but, while falling, is carried, by the orbicular motion, through 100,000 feet to $\mathrm{B}$, in the diagonal $\mathrm{EB}$.

Such a diagonal course as $\mathrm{AE}$ is therefore, in point of fact, generated by every projectile, while it is rising to any given height in the atmosphere; but, as the spectator is carried with the projectile, he merely measures its novel and peculiar motions, and is incapable of observing the orbicular motion, of which he partakes in common with the projectile. The projectile is not, howcver, the less sulject to the force of the orbicular motion, because it is not perceived or felt by a spectator at rest; but, as a body put into motion by novel forces, acting in opposing directions, its norel state of opposing motion is liable to be affected by all opposing forces simultaneously existing in nature; and, when its novel force is exhausted or destroyed at $\mathrm{E}$, it becomes the patient of the great natural forces, which, in moving the earth and atmosphere from $A$ to $B$, move it likewise, as part of the system.

The projectile having however ascended to $\mathrm{E}$, a difficulty arises in regard to the origin of the force which defiects it from the summit at $F$ towards $B$. Why does it not move for ever in the parallel DE ? What is the original force that turns it aside? Is that force required to be equal to the weight of the body; or what proportion of that force, and how is it generated ?

It appears by the fact that the whole force which was necessary to cause it to fall through the $16_{\frac{1}{12}}$ feet, is but. a $6000 \mathrm{dth}$ part of the orbicular momentum ; consequently the deflection of 16 teet forms but an angle of 20 seconds at $A$ and at $B$. The 
nascent deflection, however, at $\mathrm{E}$ would be but an infinitesimal of the said 20 seconds ; consequently, any indefinitely small deflective force, arising from new combinations of the forces, might be sufficient to return a body to the earth.

What then is the actual deflective force which turns a projectile downward, and prevents it from moving for ever in the place in the atmosphere in which it has been left by the extinction of that projectile force, which carried it from the surface into so novel a situation in the atmosphere?

Let us examine all the circumstances in which the body has been placed:-

1. It was moving with the earth in its orbit with all other lodies upon the earlh, and therefore possessed a momentum in that direction, which, with regard to other bodies, was as their guantities of matter.

2. It was defléted aside by some novel muscular or explosive force, and thrown towards $\mathrm{D}$.

3. But, while it was moving towards $\mathrm{D}$, or 16 feet and an inch, it was carried 6000 times as far, or 100,000 feet at right angles; and therefore performed a diagonal.

4. At $\mathrm{C}$ ic loses its force in the direction at $\mathrm{D}$, and is deflected towards the earih, at $\mathrm{B}$.

Let us now examine what other circumstances have attended its ascent:-

1. Its passage has been made in a resisting medium, which tended, as is knou'n, constantly to destroy the force with which it was projected from A towards $\mathrm{D}$.

2. During its ascent the point $\mathrm{A}$, and every point of the diagonal $\mathrm{AE}$, were also performing a rotary circular motion round the centre of the earih.

3. The point A would therefore be deflecled downu'ard, during its passage towards $\mathrm{C}$, from $\mathrm{A}$ below $\mathrm{C}$; so that $\mathrm{D}$ would also be deflected below $\mathrm{E}$, and the entire line $\mathrm{AE}$ would be defiected, or turned downward, below AC.

4. The rotary motion would therefore have the effect of deflecting the body below the diagonal $\Lambda \mathrm{E}$, at every increment of its ascent.

5. The constant resistance of the atmosphere would, in like manner, deflect it.

6. The body would, therefore, be sulject, during its ascent, to the action of four forces:

$a$, the orlicular force.

$b$, the projectile force.

$c$, the rotary force.

$d$, the resisting force.

7. At the apex, the projectile and resisting forces having deVol.49. Na. 230. June 1817. 


\section{The Phcenomena of Gravilation proved to be proximate}

stroyed each other, the body is then surrendered to the joint action of the orbicular force and the rotary force.

8. And we have seen that it is the effect of the rotary force to deflect it from the right line of the orbicular force towards the earth.

Consequently it is the rotation of the earth and atmosphere, acting simultaneously with the annual motion, which produces the deflection of bodies from the right lise of their orbicular motion; but the circular rotation has another important effect on the masses of various density which compose the earth, and which, as subject to a common force, would have different orbicular velocities, but for the effect of their common rotary motion in circles of different radii *.

To the rotary motion of the earth is, therefore, to be referred that uniformity in the velocity of bolies of various density, which enables the whole to keep an equal and uniform pace in the orbit of the earth. It is this rotary motion which reduces to order, what otherwise would be chaos. Hence it is that all fluids are impelled into a level surface ; hence too, doubtless, it has been, that masses of the same density have formed themselves into strata while in a state of solution; hence arise all the phænomena which result from any disturbance in the order of density ; and hence it is that, when a heavy body is thrown into lighter fluids - as air or water-the general law is proved by phænomena exactly proportioned to the relations of density.

We know, from the diurnal phanomena, that the earth and atmosphere have such a common rotary motion; and we know, from their common orbicular force, that the whole have unequal momenta. It is, however, a necessary mechanical effect of a common rotary motion, producing, of course, equal momenta if masses of various density that they range themselves, or seels to range themselves, in concentric circles, or radii of rotation, in versely, as their respective densities. The lighter bodies will be projected from the centre towards the circumference, and the

* It seems extraordinary that, a!though the two-fold niotion of the earth has so lung been known and admitted, no mechanical efiects shouid lave been considered as arising among its parts from the operation of those inotions; and that one motion should have been considered as laving no ocher end than the alternation of seasons, and the other none but the changes of day and night. It is true that these are some of the ends resulting from the two-fold motion; but gencral causes have many effects, and these are rather consequential than proximate. The proximate effect of motion is force; the proximate effect of force is the communcation of motion, force, anl momentum: and in this instance the motions of the earth produce in the.parts motion, force, and momenta: which, diverted, deflected, or turned aside, by any foreign inuscular, mechanical, or explosive force, producc the phænomena of resistance and wciglat. 
heavy ones will be forced towards the centre by their mutual collisions. Such will be the law, governing all the masses that are free, to move one among another, as the fluid parts and the moving or moveable parts. It is a law growing out of the conditions, and the conditions are those which exist in Nature. If a projectile, therefore, have a specific density equal to the air or fluid into which it has risen, it will be carried round the earth in the concentric circle of that stratum, because the momenta are there equal ; but, if it be lighter than the air, it will then be reflected by the denser strata, till its own momentum, and the momenta of the surrounding air, be equal; while, on the other hand, if it be heavier than the circumambient air or fluid, then the air or fluid will rise over it and deflect it to the earth, with a force which will be nearly in the ratio of their densities.

Galileo was the first geometrician who analysed the phænomena of falling bodies, and determined the law of their motions. He was followed in this theory by Newton, and his doctrines are justly respected to this day. 'That great man considered, however, that bodies thrown perpendicularly upward, merely describe in rising and falling the same straight line. He was one of the most powerful advocates of the two-fold motions of the earth; yet he never considered bodies acted upon by a temporary and relative projectile force, as still subject to the absolute two-fold motions of the earth and atmosphere. Hence he considered the force which returned the body to the earth, as equal in small distances to the weight of the body, and as acting in right lines from the centre of the earth. In this notion he was borne out. by the belief in all kinds of sympathies and emanations which characterized his age, as well as that of Newton; and to these superstitions may, doubtless, be referred the doctrine of an emanating gravity. It appears, however, that, as a body subject to the novel force of an upright projectile, does not, in truth, describe a perpendicular line, but two sides of an exceedingly obtuse triangle; no force is requisite to deflect it to the earth, but the exceedingly small one which creates the nascent deflection. Hence, as the angle of deflection required to carry a body through the first inch, is not the $2000 \mathrm{dth}$ part of a degree; the deflective force need not be more than the $120,000 \mathrm{dth}$ part of the permanent momentum of the body created by the orbicular force: and consequently no difficulty arises in referring the small deflective force to the combinations of the great motions of our planet. 


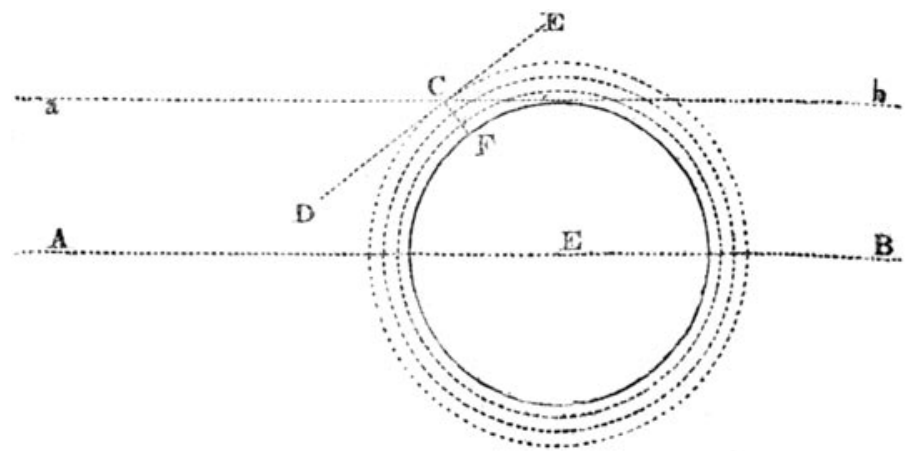

Suppose $E$ to be the earth; $A B$ a portion of its orbit ; $F$ a place on the earth's surface; $\mathrm{C}$ a stratum of the atmosphere. which a projectile has reached; $a b$ a parallel of the orbit, and $\mathrm{DE}$ a tangent of the atmosphere; then it is evident that, whether the earth is turning from $D$ towards $\mathrm{E}$, or fitont $\mathrm{E}$ towards $\mathrm{D}$, a deflection, from the orbicular motion to the rotary, would take place, measured by such an angle as $\mathrm{DC} a$, or $l \mathrm{CE}$, in either case creating a direction of force opposed to that in the orhit, and producing a resistance in the next superior atmosphere, and creating a defection downward fully equal to the known phrenomena, which requires for the first deflection but an infinitely small portion of an angle of 20 seconds.

It is also evident that the common rotary motion of the earth and atmosphere would confer a greater velocity on the circle $\mathrm{C}$ than the circle $F$, and so in every circle from $C$ to $F$, the spaces described being as the squares of the radii, or as the squares of the distances from $E$. To confer, however, an equal mon:enturan on the mass, it is necessary that the densities from $\mathrm{C}$ to $\mathrm{E}$ shonla be inversely as the velocity, or inversely as the spaces, or inversely as the squares of the radii.

Moreover, as by the phanomena, the momenta in every concelitric stratum or circle of rotation are known to be equal, so any mass of equal density would in different circles, or at differezat distances, be carried or impelled in the vortex by the circumambient media, or incumbent bodies, through spaces, which would be to each other, inversely as the squares of the distunce from the centre, and directly as the resistance of the medium in which they move.

Consequently, the collision of the bodies or masses of different densities, in the terrestrial system, will force or urge the heavy bodies towards the centre, and of course, also, the light ones towards the circumfercise-circumstances which we know accord 


\section{Effects of the Orlicular and Rotary Motions of the Earth. 437}

with the phænomena of all fluid bodies, and of all fixed bodies when deprived of competent support, and of all bodies moved out of their station by muscular or explosive force, and left without support. Q. B. D.

The following illustrations and observations may tend to make these principles perfectly clear, and to remove all douhts.

1. The projectile at its apex, when deprived of the projectile force, is still immersed within the atmosphere, and is carried, or urged, by the atmosphere, in the direction of the circular rotation of the atmosphere.

2 . 'That circular rotation then produces, or solicits to produce, (with a force proportioned to the relative densities of the atmosphere and projectile,) a deflection of the said projectile from the right line of the orbicular motion, into the direction of the eircular motion at the place of contact.

3. If the density of the projectile were equal to the density of the atmosphere, then the projectile would float in the atmosphere, and be carried round the earth in the circular vortex of the earth, like the atmosphere itself.

4. If it were denser than the atmosphere, then the rotary momentum of the lighter atmosphere being less than the rotary monentum of the projectile, resistance would be generated equal to the difference of their rotary momenta; and a deflection of the denser body by the lighter atmosphere, into a sinaller circle of rotation, would take place till it reached a competent basis on the earth.

5. If the projectile were lighter than the atmosphere, then the momentum of the circumjacent atmosphere would be greater than that of the projectile, and the projectile would, in consequence, be forced upward into a larger rotary circuit, till its rotary momentum equalled that of the circumjacent atmosphere.

$\dot{6}$. In media of uniform density, as in water, the projectile, if heavier, sinks to the bottom ; if lighter, floats on the top, equalizing its monentum by presenting a portion of its mass within the air; or, if of the same weight, floats indifferently within the fluid.

7. As the deflective force is continually operating during the fall, and as every uniformly continued force generates a constantly accelerated motion, so as bodies fall by the action of a continued deflective force, they are necessarily accelerated during their fall, till they rest on the fixed parts of the earth. But, as is found by experiment, the increased resistance of the air, arising from the increased motion, will oceasion a balance of the two forces, and an equable motion during part of the fall.

S. Combining the time of falling, the spaces as determined by Galileo, and by alleged experiments, with the actual bases generated by the orbicular motion, the following will be the eleE e 3 ments 


\section{8}

The Phonomena of Gravitation proved to le proximale

ments of bodies falling in times between four seconds and the tenth of a second:

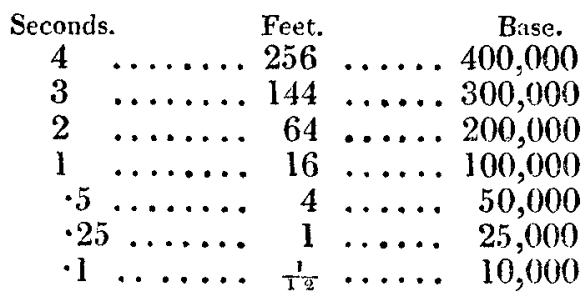

9. As every successive circle, from the centre to the utmost bounds of the atmosphere, in performing the diurnal rotation, contains bodies of such density, that the density multiplied by the motion is equal ; so no body of undue density would remain in a circle of rotation which created in it too great or too small a relative momentum, provided, as in fluids, the parts were free to move one among another; and, if they were not free to move, as in fixed organizations, then they would exhibit the phrnomena of pressure, or would appear, in regard to other bodies, to solicit to ascend or descend, as the case might be. Probably to this conflict of light and heavy bodies, to the generation of light bodies in undue positions, and to the varied action of fixed and fluid matter, may be referred most of the phænomena of terrestrial organizations; and, as the whole grows out of the two-fold motion of the earth, so we thus refer the origin of ail things and phænomena to the agency of motion.

It follows, therefore, that, the velocity of all the parts and dependencies of the earth being alike, the momentum of every part is directly as its quantity of matter; consequently, MOMENTUM Is WEIGHT; and the velocity of 100,000 feet per second in the direction of the tangente of the orbit being so nuch greater than the ordinary, or even extraordinary forces applied to move bodies in any novel direction, the phrnomenon called by the name of Gravitation is a necessary result of a preponderating, coincident motion in another direction, all contrary or foreign motions being ultimately destroyed by the resisting medium of the atmosphere and the deflective circular motion.

The weight of bodies, then, acts on the same principle as their momentum in the orbit of the earth, because every force whicin exhinits the phæ: omena of weight affects the paramount orbicular force; and, thi, active principle is, therefore, merely a phan menom tewling from the orbicular momentum, regulated by the rotary motion; which rotary motion causes all bodies to perforn circuits inversely as their quantities of matter, and which circuits are proportioned to the distance from the centre.

The phænomena of weight and gravitation, and of falling bodies, 


\section{Effects of the Orbicular and Rotary Motions of the Earth. 439}

bodies, mav therefore be considered as a result of the composition of forces produced by the annual and diurnal motions of the earth and its adjuncts. Of course, ail the motions and laws of projectiles are subordinate to these, and are included within them.

On a small scale, the principle may be illustrated by experiments of analogons phronomena, in which it will appear that any lateral motion of bodies produces the same results as the earth itself ; and that, as a consequence of every such lateral motion, the projectiles from such hodies respect, in falling, the bodies whence they are projected, though no suspicion or allegation of attraction can, in such cases, be adduced.

Thus, as it is well known, a ball projected from a ship in motion falls at the place whence it rose, though, during its ascent and descent, the ship proceeded many yards. The ball, though detached from the ship, respected the ship, during its flight and fall, exactly as though it had been attracted by the ship. It cannot, however, be contended that such phænomena are results of the ship's attraction! The effect arises merely from the ship's lateral motion having been acquired by the ball previously to its projection; the case being exactly the same as that of a ball projected above the earth, to which it falls again, on the very principle on which it fell on the ship, not by the attraction either of the ship or the earth, but because the earth's lateral motion, like the lateral motion of the ship, had been acquired by the ball.

So, also, when a man, during feats of horsemanship, throws up oranges in his course, he readily catches them again, though during their flight he advanced many yards, becatse his lateral motion had previously been given to the oranges; and in consequence, in falling again, they respected him just as though, according to pre-existing notions about the earth, he had attracted them.

In like manner, if a ball be dropped from the top of the mast of a ship, which at the time was moving at any given rate, the ball will fall perpendicularly at the foot of the mast, exactly as though the ship had been standing still; and will thus appear to be infuenced by the ship, and not by the earth, exactly as a ball let to fall from a tower falls to the earth, and appears to respect the earth. In both cases, the phænomena are regular and necessary consequences of the composition of motion; and there is no unseen or occult power, called attraction, concerned in one case more than in the other. 
ILLUSTRATION.

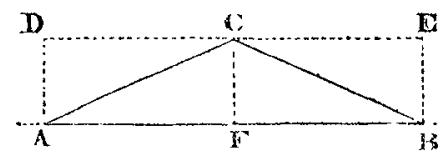

If a ball be projected from A perpendicularly towards $D$, and $\Lambda$ be supposed to be on the deck of a ship, which is moving at any given rate, from the part $A$ towards the part $B$; then the ball, instead of ascending to the point of space at $D$, will, without material error, move in the diagonal $\mathrm{AC}$, by the compound forces which, in the same time, would have carried it to D or F; and in descending it will fall to the deck at $B$, to which the point $A$, in the interim, has advanced. It has in truth performed, instead of an apparent perpendicular, the two sides of the triangle, or the curve $A C B$; and instead of falling at the point of space $A$, whence it was projected, it has advanced with the ship, and fallen at the point of space $B$, to which $A$ has advanced; and in falling has respected the ship, and not the point of space, or the earth at A. It was not however attracted by the ship, but mercly impelled in the diagonal by the motion of the ship, in which it previously participated.

These cases fully illustrate the principle for which I contend; but, of course, the relations and powers of the ship, and mon moving on the moving earth, are included within the more extensive relations and powers operating in Nature, in the prodigious forces generated by the annual and dinrnal motions of our planet. The case of a body thrown upward from a ship in motion, merely shows that, in falling, it obeys a law growing out of the motion of the ship; in like manner as bodies projected from the earth, or falling towards it, obey a similar law of motion growing out of similar motions of the earth. The law in both cases is a mere result of the composition of motion, and not an effect of any occult or mysterious power.

These examples, and others that might be adduced, serve, however, to prove the exact analogy of the powers, the phænomena, and the results. No experiments in physical philosophy seem to afford more conclusive analogical proofs of doctrines relative to any natural operations, which are too vast to be vieved by man on the: great scale.

The recognition of the principles of this paper may be expected to lead to more precision in the doctrines of projectiles. Many incomprehensible irregularities in Dr. Hutton's and Mr. Robins's experiments on gunnery may be found to arise from the contrariety, opposition, or nentrality, of the direction of the ro- 
sary motion, in relation to that of the orbicular motion. It will likewise be found that periodical fuctuations in the weight, and deflections of the atmosphere and other fluids, may be traced to periodical variations in the relative directions of the mechanical forces, as in the tides, monsoons, trade-winds, \&c.

To extend these principles to the solution of the phænomenn of Nature, and to deduce from them all the results of which they are susceptible, would be, in regard at least to nomenclature, to remodel the "Principia" of Newton, and to arrange a new system of physics.

Nevertheless, I feel it proper to state, that these demonstrations of the true and necessary causes of the phanomena hitherto ascribed to an unknown power, called by the name of Gravitation, merely fill some important connecting terms in the series of mechanical causes ascertained by modern philosoplyy; while they disturb none of the known relations of bodies, as determined by experiment and observation, or by the geometrical and analytical investigations of Galileo, Kepler, Descartes, Newton, Euler, La Grangè, Herschel, or Laplace.

A Summary of these doctrines may, perhaps, without material error, or cmission, be expressed in the following paragraphs :

1. That bodies moved in the annual orbit of any planet, acquire a momentum in the direction of the increments of that orbit superior to the influence of any other permanent force which is communicated to them.

2. That all variations in the direction of this orbicular motion are effected by deflections of that paramount motion, either by the rotary diurnal motion, or by some muscular, mechanical, or chemical force.

3. That the resistance which bodies exhibit in being lifted, or thrown, or in any way turned into any new direction, is the neasure of their weight, which is as their quantity of matter, because it arises from a velocity common to the terrestrial system in the direction of the orbit.

4. That the phænomena of falling bodies are produced by the deflection of the circular rotary motion from the comparatively straight line of the orbicular motion.

5. That every body which has had any new direction of force given to it, is nevertheless subject to the permanent influence of the pre-existing orbicular and rotary forces in the lines of their direction, and the resulting line of motion is the effect of all the operative forces.

6. That the phænomenon of the ascent and descent of bodies is also influenced by the resistance of the medium through which its novel direction of motion has forced it to proceed.

7. The force with which the deflection by the rotary motion 
is produced, is as the density of the body deflected to the density of the medium in which it moves, and in the inverse ratio of the squares of the distance from the centre.

8. That it is the necessary tendency of the rotary motion to give an equal momentum to the heterogeneous masses composing a planet and its atmosphere, while the whole are moving with an equal velocity in the orbit.

9. 'That all phænomena of motion visible to beings who partake of the common motions of a planetary system, are either the relative motions of distant orbs, or the disturbance of the great common motions by new directions of motion produced by some muscular, mechanical, explosive, or chemical force.

10. That these temporary and novel directions of force and motion are speedily extinguished by the great permanent forces moving in other directions, all traceable to the phrnomena, and producing compositions of motion which result from the known laws of dynamies.

11. Tliat of course similar motions produce similar phænomena in all planetary bodies.

12. That, therefore, the phænomenon hitherto ascribed to an nccult power called gravitation, is a simple result of known motions.

The application of these principles to the phænomena of a system of bodies moving within the gaseous medium of universal space, will be the object of some future paper.

Holloway, June 10, 1817.

CI. On Mr. Liston's, or the Euharmonic Scale of $M u-$ sical Intervals, extended according to his tuning Process, from 59 to 612 Notes in the Octave; showing thus, a Division of the Octave into 612 equal Parts, or as nearly so, thronghout, as Experiments in Harmonics, or the most refined musical Performances, seem to require.

By $M r$. John Farex, Sen.

To Mr. Tilloch.

SrR, - Ir has been truly remarked, that, " $_{\mathrm{T}}$ By stopping in the investigation of any subject, short of its thorough or general investigation, we are liable to fall into the error, of mistaking particular cases, for general facts, and of too soon drawing conclusions, which on more matued consideration, require to be recalled or amended." Thus it has in a small degree happened, with regard to one of the positions respecting the Euharmonic or Listonian Scale of Musical Intervals, in my paper on the Nomenclature of these Intervals, which you did me the favour to insert 